\title{
Morfometría de las especies de Vieja (Cichlidae) en ríos de la cuenca del Usumacinta, Chiapas, México
}

\section{Morphometry of Vieja species (Cichlidae) in rivers of Usumacinta's basin, Chiapas, Mexico}

\author{
Miriam Soria-Barreto ${ }^{1,2 *}$, Rocío Rodiles-Hernández y Alfonso A. González-Díaz ${ }^{1,2}$ \\ ${ }^{\prime}$ El Colegio de la Frontera Sur. Carretera Panamericana y Periférico Sur s/n, Apartado postal 63, 29290 San Cristóbal de Las Casas, Chiapas, México. \\ ${ }^{2}$ Dirección actual: Unidad Académica de Agricultura, Universidad Autónoma de Nayarit. Km. 9 Carretera Tepic-Compostela, 63780, Xalisco, Nayarit, \\ México. \\ *Correspondencia: mmsoriab@yahoo.com.mx
}

\begin{abstract}
Resumen. Se evaluaron y compararon diversas características morfológicas de 6 especies de Vieja Fernández-Yépez, 1969 del río Usumacinta. Se emplearon 20 organismos adultos por especie y 33 medidas corporales que fueron estandarizadas y examinadas con un análisis discriminante. Además se comparó la forma del cuerpo entre las especies, mediante el análisis de morfometría geométrica a partir de imágenes digitalizadas. El análisis con la morfometría tradicional y geométrica permitió diferenciar los seis taxones. Se encontraron 10 medidas discriminantes, que expresan diferencias significativas en la longitud de las aletas y la región cefálica, de las cuales la posición de la boca diferenció estadísticamente más taxones. Las relaciones de similitud con ambos análisis mostraron 2 grupos, siendo $V$. bifasciata (Steindachner, 1864) y V. synspila (Hubbs, 1935) las especies más parecidas. Las gradillas de deformación mostraron que la mayor variación está en la región cefálica, específicamente en la posición de la boca y los ojos. Las diferencias morfométricas encontradas pueden servir para la determinación taxonómica en trabajos futuros. La variación encontrada en la morfología cefálica y trófica puede ser un mecanismo que favorece la coexistencia de las especies y la diversidad del grupo en la región.
\end{abstract}

Palabras clave: peces, cíclidos, taxonomía, morfometría geométrica, morfometría tradicional.

\begin{abstract}
We evaluated and compared morphological characteristics among the 6 species of Vieja Fernández-Yépez, 1969 from Usumacinta River. We analyzed 20 adults by specie, obtaining 33 measurements, which were standardized and analyzed with a discriminant analysis. We also performed a comparison of body shape among species, using the geometric morphometric analysis from digitalized images. Traditional and geometric morphometrics allowed distinguishing each taxa. We found 10 discriminants measures, related with the head region and length of fins, only mouth position was different among more taxa. In both analyses, similarity relationships showed 2 groups, being $V$. bifasciata (Steindachner, 1864) and V. synspila (Hubbs, 1935) the most similar species. Thin plate spline showed greater variation in the cephalic region, specifically in eyes and mouth position. Morphometrics differences can be used for taxonomic identification in future works. The variation found in cephalic and trophic morphology can be a mechanism that promotes coexistence of species and explain the diversity of the group in this region.
\end{abstract}

Key words: fishes, cichlids, taxonomy, geometric morphometrics, traditional morphometrics.

\section{Introducción}

En México, la familia Cichlidae constituye el segundo grupo de peces dulceacuícolas más diverso; está constituido por 57 especies cuya distribución abarca desde el río Hondo hasta el río Bravo, en la vertiente del Golfo y desde el río Suchiate hasta el río Yaqui, en el Pacífico; sin embargo, su mayor diversidad se ubica en el sureste mexicano (Miller et al., 2005). Específicamente, la cuenca

Recibido: 11 septiembre 2009; aceptado: 10 agosto 2010 del río Grijalva-Usumacinta es el sistema hidrológico con más cíclidos nativos y endémicos (Miller, 1986), donde se siguen registrando organismos cuyo estatus taxonómico es incierto (González-Díaz et al., 2008) y nuevos taxones como Rocio ocotal Schmitter-Soto, 2007.

La clasificación de los cíclidos mexicanos ha cambiado y sigue modificándose. Inicialmente las especies fueron ubicadas en Cichlasoma Swainson, 1839 (excepto el monoespecífico Petenia Günther, 1862). No obstante, la revisión de Kullander (1983) restringió el género a 12 especies de Sudamérica y para evitar confusiones propuso que los cíclidos centroamericanos se citaran entre comillas 'Cichlasoma', hasta que su taxonomía fuera revisada. Ade- 
más, sugirió que en ciertos taxones podrían emplearse las secciones de Regan (1905) en el nivel de género.

A la fecha, mediante el uso de análisis filogenéticos se ha logrado precisar el estatus taxonómico de algunos géneros como Herichthys Baird y Girard, 1854 (Hulsey et al., 2004) y Thorichthys Meek, 1904 (Roe et al., 1997). Por el contrario, otros cíclidos como aquellos incluidos en el género Vieja Fernández-Yépez, 1969, ameritan una exhaustiva revisión taxonómica.

Este taxón está conformado por 17 especies, distribuidas en Centroamérica y México (Kullander, 2003; Miller et al., 2005). Su nomenclatura ha cambiado a través del tiempo. En un principio sus especies fueron designadas a Cichlasoma; posterior a la revisión de Kullander quedaron en 'Cichlasoma' y después fueron reasignadas a Vieja (Kullander, 2003). Actualmente no es claro su estatus taxonómico, entre otra cosas porque se ha sugerido que es un grupo polifilético (Miller et al., 2005).

La diagnosis del género por Fernández-Yépez se basó en la forma del cuerpo, fue definido por peces de cuerpos altos y el pedúnculo caudal corto y alto, con dientes bicúspides (citado en Allgayer, 1989). No obstante en el grupo se incluyen especies con características distintas, peces con cuerpos alargados y pedúnculos largos o con dientes aplanados o cónicos, por lo cual han sido son reubicados en diferentes géneros. Otro criterio comúnmente usado en la determinación es el patrón de coloración, que puede variar desde bandas laterales o verticales. De este modo, las especies se han asignado a Paraneetroplus Regan, 1905, Paratheraps Werner y Stawikowski, 1987, Theraps Günther, 1862 o simplemente 'Cichlasoma' (Conkel, 1993; Hulsey et al., 2004; Concheiro et al., 2007; Rícan et al., 2008; Eschmeyer y Fricke, 2010; Froese y Pauly, 2010; López-Fernández, com. pers.).

La determinación taxonómica de las especies de Vieja usando ejemplares de colecciones, en ocasiones resulta difícil y confusa, por la falta de caracteres discriminantes en algunos taxones o debido la semejanza morfológica en especies simpátricas y donde sólo el patrón de coloración permite su diferenciación. Por ello es necesario profundizar en aspectos básicos de la taxonomía de este grupo de cíclidos, buscar más y nuevas características morfológicas que ayuden a conocer, clasificar y explicar su diversidad en la región.

La diferenciación entre especies relacionadas y semejantes puede lograrse con el análisis morfométrico. Tradicionalmente se han empleado las medidas morfológicas para la descripción taxonómica, evaluar la variación morfológica, definir medidas discriminantes y establecer relaciones de parentesco. Sin embargo, en tiempos recientes la morfometría geométrica ha sido empleada como una herramienta para describir y comparar la morfología de taxones relacionados (Adams et al., 2004; Zelditch et al., 2004). Esta técnica de análisis considera la morfología completa y la variación intraespecífica, disminuye la variación producto del tamaño corporal; sus resultados se han utilizado para hacer interpretaciones desde el punto de vista funcional e inferir procesos evolutivos.

De este modo, los objetivos del presente trabajo son conocer y comparar las características morfométricas tradicionales de las especies de Vieja presentes en el Río Usumacinta: $V$. argentea Allgayer, 1991, V. bifasciata (Steindachner, 1864), V. intermedia (Günther, 1862), V. pearsei (Hubbs, 1936), V. synspila (Hubbs, 1935) y $V$. ufermanni Allgayer, 2002. Encontrar características morfométricas útiles para el reconocimiento de los taxones, así como conocer la variación en la forma del cuerpo a través de la morfometría geométrica.

\section{Materiales y métodos}

Se analizaron las especies de Vieja procedentes del río Tzendales y Lacanjá en Chiapas. Estos ríos son tributarios del río Lacantún y forman parte de la región hidrológica del Usumacinta. Se localizan en la Reservas de la Biosfera Montes Azules y Lacantún en la región de la selva lacandona en México (Rodiles-Hernández et al., 1999; INE, 2000).

El material biológico se obtuvo de la Colección Ictiológica de ECOSUR-San Cristóbal (ECOSC), su identidad taxonómica fue corroborada con las claves de Miller et al. (2005) y las descripciones de Allgayer (2002). Se analizaron 20 ejemplares por especie con los siguientes números de catálogo. Vieja argentea: ECOSC 2558, 4685, 4694, 4701, 4709, 4716, 4747, 4750, 4806, 4815, 4821, 4823, 4881 y 4886. Vieja bifasciata. ECOSC 859, 1794, 2673, 2675, 2678, 2708, 2709, 2762, 3874, 4473, 4675, 4677, $4689,4698,4704,4746$ y 4880. Vieja intermedia: ECOSC 4684, 4706, 4710, 4723, 4726, 4728, 4730, 4738, 4742 y 4743. Vieja pearsei: ECOSC 102, 1712, 2318, 2352, 2508, 2547, 4118, 4696, 4740, 4748, 4749, 4812, 4820, 4875 у 4878. Vieja synspila: ECOSC P 4697, 4703, 4712, 4713, 4722, 4724, 4745, 4804, 4805, 4883, 4889 y 4893. Vieja ufermanni: ECOSC 134, 186, 409, 1729, 2298, 3868, 4678, 4687, 4693, 4708, 4741, 4874 y 4894.

Se seleccionaron ejemplares adultos, los criterios para considerarlos como adultos fue la talla de primera madurez reportada por Konings (1989) y Chávez-Lomelí et al. (1988) o indicios de actividad reproductiva. También se consideró la condición de fijación, es decir que los especimenes no estuvieran torcidos o con la boca abierta.

Las medidas tradicionales se obtuvieron con el empleo 
de un vernier electrónico con precisión de $0.01 \mathrm{~mm}$, se tomaron las siguientes: (1) longitud estándar, (2) longitud cefálica, (3) altura máxima de la cabeza, (4) diámetro ocular, (5) longitud del hocico cerrado, (6) longitud de la mandíbula superior, (7) longitud de la mandíbula inferior, (8) abertura bucal, (9) altura de la mejilla, (10) ancho de la cabeza, (11) distancia interorbital, (12) distancia predorsal, (13) altura máxima del cuerpo, (14) ancho del cuerpo, (15) distancia interpectoral, (16) altura del pedúnculo caudal, (17) longitud del pedúnculo caudal, (18) longitud de la aleta dorsal, (19) altura de la aleta dorsal, (20) longitud de la aleta anal, (21) altura de la aleta anal, (22) longitud de la aleta caudal, (23) altura de la aleta caudal, (24) longitud de la aleta pectoral, (25) longitud de la aleta pélvica, (26) posición del ojo, (27) longitud del hocico abierto, (28) ancho de la boca abierta, (29) ancho de la boca cerrada, (30) altura bajo la línea media, (31) ancho del pedúnculo caudal. Además se obtuvo el (32) ángulo formado por las mandíbulas y (33) la posición de la boca. De acuerdo con los criterios de Meyer (1987) y Winemiller (1991).

Las primeras 25 medidas fueron expresadas en proporción de la longitud estándar. La medida de la posición del ojo (26) fue dividida entre la altura cefálica (3); la altura de línea media (30) entre la altura corporal (13). Se obtuvo el valor de la expansión del hocico dividiendo la medida del hocico abierto (27) entre el hocico cerrado (5); así como la proporción entre el ancho de la boca abierta (28) y la boca cerrada (29). Se calculó el aplanamiento del pedúnculo al dividir la altura del pedúnculo (16) en la parte media y el ancho del mismo (31). Estas transformaciones se realizaron para reducir el efecto alométrico causado por las diferencias de tamaño entre los individuos (Crisci y López Armengol, 1983). A continuación, todas las medidas y los 2 ángulos fueron transformados en logaritmo base 10 .

Se realizó un análisis discriminante, con la finalidad de reconocer las medidas más importantes para separar las especies y conocer patrones de variación morfológica. Se calcularon los 2 primeros factores, que explican la mayor varianza y se consideró que las medidas más importantes para predecir los grupos, tenían valores absolutos superiores a 0.3 (Catena et al., 2003). A estas medidas, se le aplicó un análisis de varianza y una prueba de DHS Tukey, con la finalidad de confirmar las diferencias significativas entre las especies, con el empleo del programa Statistica ver.7.0 (StatSoft, 1995).

Las relaciones de similitud morfológica se obtuvieron con un análisis de agrupamiento, se usó la matriz de distancia cuadrática de Mahalanobis obtenida del análisis discriminante; el dendrograma se representó con el método de ligamiento promedio (UPGMA) en el programa Statistica ver. 7.0 (StatSoft, 1995).

Para el análisis de morfometría geométrica, se tomaron imágenes del lado izquierdo de todos los ejemplares de cada una de las especies con una cámara digital (Sony DSC-S40). Las imágenes se digitalizaron mediante el programa TPSdig (Rohlf, 2004) y se ubicaron 15 marcas (landmarks) en los siguientes sitios (Fig. 1): 1, punta de la maxila superior; 2, inserción anterior de la aleta dorsal; 3 , inserción posterior de la aleta dorsal; 4, inserción dorsal de la aleta caudal; 5 , inserción media de la aleta caudal; 6 , inserción ventral de la aleta caudal; 7, inserción posterior de la aleta anal; 8, inserción anterior de la aleta anal; 9,

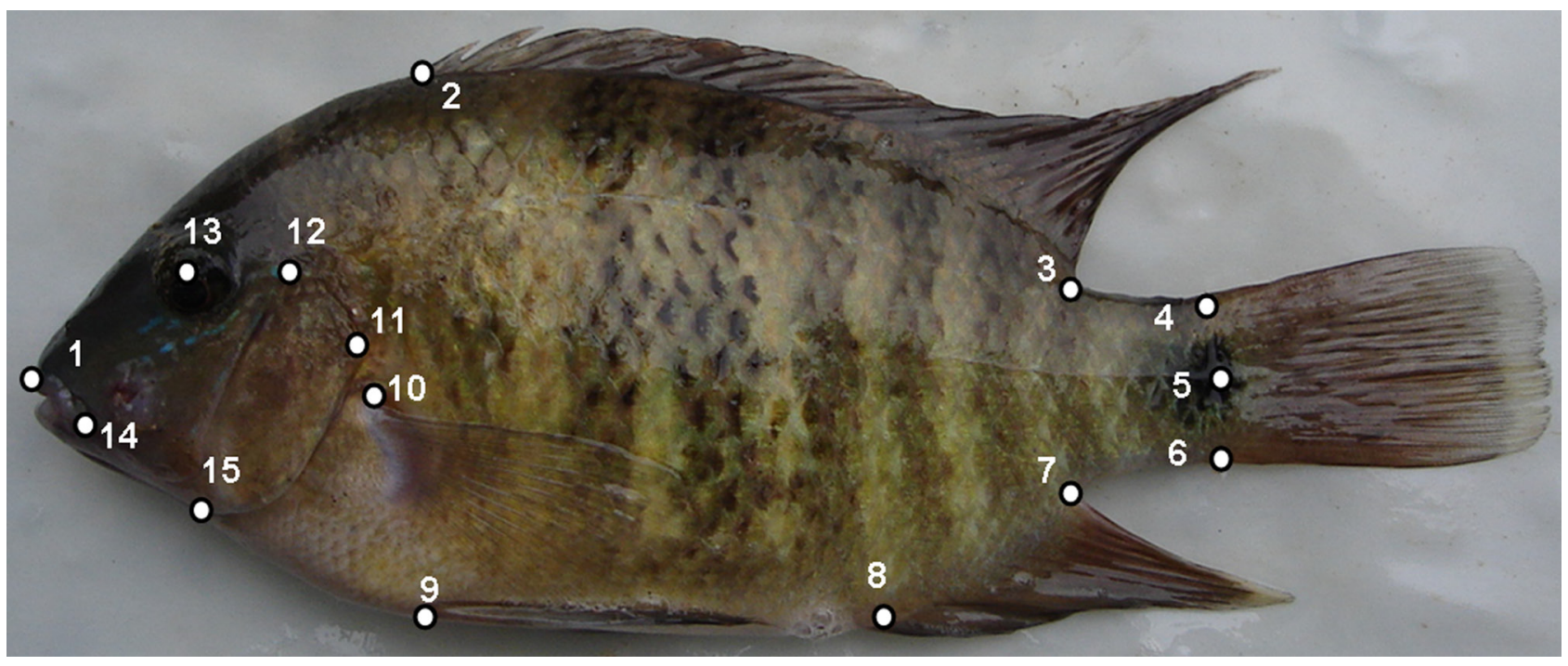

Figura 1. Ubicación de las marcas usadas para el análisis de morfometría geométrica. 
inserción de la aleta pélvica; 10 , inserción de la aleta pectoral; 11, borde posterior del opérculo; 12 , borde dorsal del preopérculo; 13, punto medio del ojo; 14, borde posterior del maxilar y 15, margen anterior del cleitro.

Las marcas de todos los organismos fueron alineadas y reescaladas mediante el análisis generalizado de Procrustes (Rohlf, 1999), el cual elimina la información que no está relacionada con la forma tal como la variación en la posición de los ejemplares. Los valores de las deformaciones parciales (partial warps) se emplearon en un análisis de discriminantes con el programa CVAGen6m del programa IMP; con el propósito de evaluar y comparar la morfología entre los taxones.

La visualización de la forma del cuerpo entre los cíclidos se realizó con un análisis de deformación (thin-plate spline) en el programa IMP (Sheets, 2001). Este análisis emplea la morfología de todos los organismos para obtener una forma promedio, a partir de la cual se compara la posición de las marcas, así las diferencias se representan como una gradilla deformada. Cabe señalar que en el presente trabajo estas figuras se generaron a partir del gráfico de análisis de discriminantes, las deformaciones han sido exageradas 3 veces para tener una mejor percepción de las diferencias morfológicas y que los cambios mostrados correspondan al promedio de cada especie.

A partir del análisis de discriminantes se generó la matriz de distancias cuadráticas de Mahalanobis; dicha información sirvió para elaborar un dendrograma utilizando el método de ligamiento UPGMA, con la finalidad de establecer las relaciones de similitud entre las formas.

\section{Resultados}

El análisis de discriminantes con las medidas tradicionales mostró que los 2 primeros ejes son estadísticamente diferentes (Lambda de Wilk's $=0.00008$, F $(155,420)=15.731, p<0.00001)$. La primera función canónica explicó un $38.1 \%$ de la varianza, se diferencia en el extremo positivo a $V$. argentea, $V$. bifasciata y $V$. synspila por tener valores altos de la longitud de la aleta anal, la altura y longitud cefálica, la posición del ojo y boca. En la región negativa están el resto de las especies, caracterizadas por tener más largo el hocico y la aleta pélvica, cabezas anchas, cuerpos más altos y mayor altura de la línea media.

La segunda función explicó el $28 \%$ de la variación; en la región positiva se ubica $V$. argentea $y$ V. intermedia al poseer mayor distancia predorsal, longitud de la mandíbula superior, ancho del cuerpo y longitud de la aleta dorsal, así como el ojo y la boca en posición más dorsal y el pedúnculo más alto y delgado. Hacia la región negativa, las especies se diferencian por tener mayor diámetro ocular, longitud de la aleta anal, caudal y el hocico (Fig. 2, Cuadro 1).

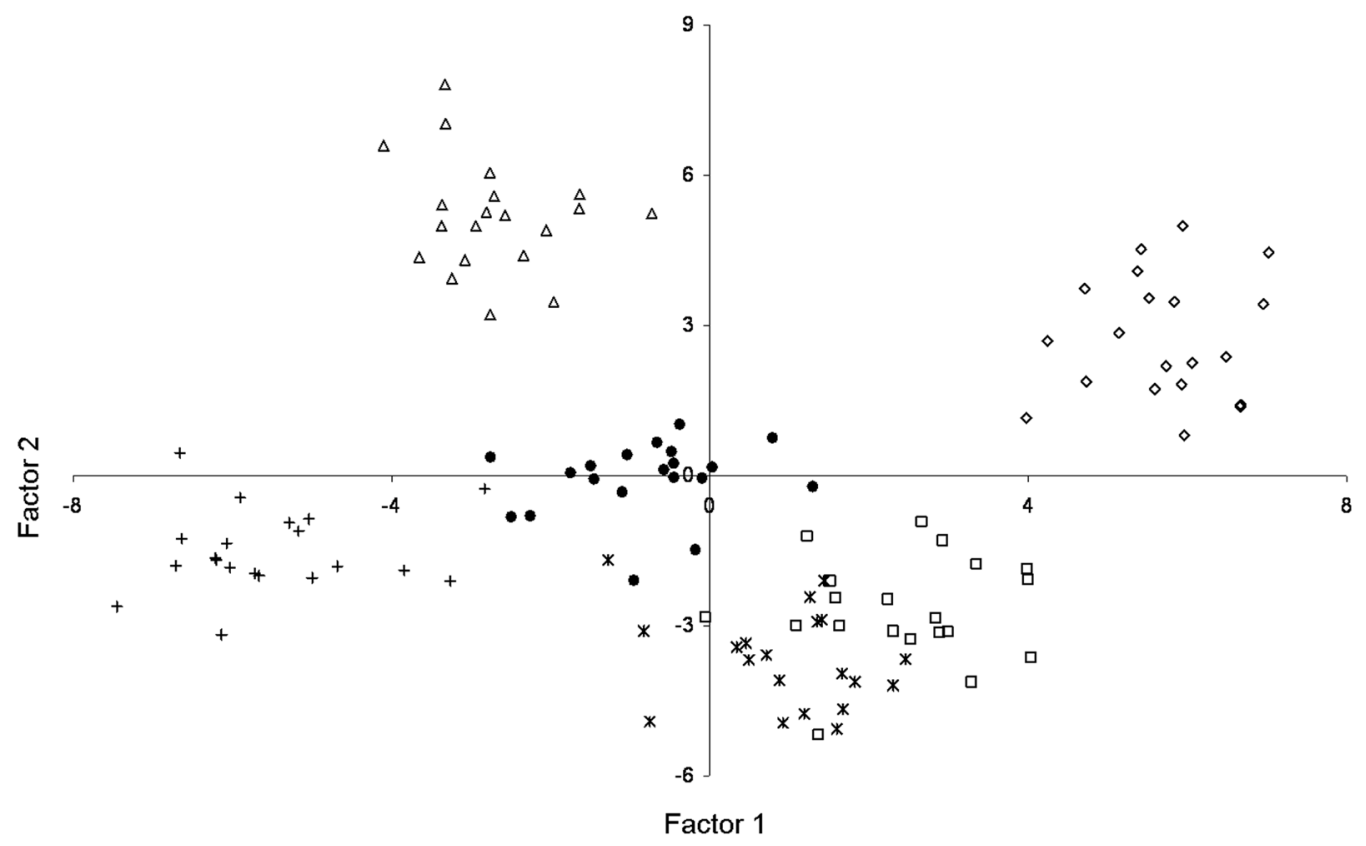

$\diamond V$. argentea $\square$ V. bifasciata $\Delta V$. intermedia $+V$. pearsei $* V$. synspila $\bullet V$. ufemanni

Figura 2. Análisis de discriminantes con medidas tradicionales en las especies de Vieja. 
Cuadro 1. Matriz de coeficientes de los análisis de discriminantes en las especies de Vieja

\begin{tabular}{|c|c|c|}
\hline Medidas & Función 1 & Función 2 \\
\hline Longitud cefálica & 0.321 & -0.232 \\
\hline Altura cefálica & 0.637 & 0.181 \\
\hline Diámetro ocular & 0.127 & -0.365 \\
\hline Longitud del hocico & -0.349 & -0.647 \\
\hline Longitud de la mandíbula superior & -0.022 & 0.431 \\
\hline Longitud de la mandíbula inferior & 0.154 & 0.235 \\
\hline Altura de la boca & 0.029 & -0.199 \\
\hline Altura de la mejilla & -0.162 & -0.207 \\
\hline Ancho de la cabeza & -0.364 & -0.086 \\
\hline Distancia interorbital & -0.179 & -0.085 \\
\hline Distancia predorsal & 0.174 & 0.551 \\
\hline Altura corporal & -0.363 & -0.265 \\
\hline Ancho del cuerpo & 0.203 & 0.327 \\
\hline Distancia interpectoral & -0.092 & 0.058 \\
\hline Altura del pedúnculo caudal & 0.291 & -0.292 \\
\hline Longitud del pedúnculo caudal & 0.026 & 0.015 \\
\hline Longitud de la aleta dorsal & -0.295 & 0.310 \\
\hline Altura de la aleta dorsal & -0.159 & -0.272 \\
\hline Longitud de la aleta anal & 0.913 & -0.371 \\
\hline Altura de la aleta anal & 0.109 & -0.116 \\
\hline Longitud de la aleta caudal & 0.228 & -0.633 \\
\hline Altura de la aleta caudal & -0.075 & 0.238 \\
\hline Longitud de la aleta pectoral & 0.004 & 0.177 \\
\hline Longitud de la aleta pélvica & -0.453 & 0.136 \\
\hline Posición del ojo & 0.626 & 0.462 \\
\hline Expansión del hocico & -0.133 & -0.044 \\
\hline Proporción de la boca & -0.083 & 0.005 \\
\hline Altura bajo la línea media & -0.343 & 0.267 \\
\hline Aplanamiento del pedúnculo & -0.294 & 0.369 \\
\hline Ángulo de las mandíbulas & -0.048 & -0.055 \\
\hline Posición de la boca & 0.561 & 0.457 \\
\hline
\end{tabular}

Con negritas, los eigenvalores de las medidas más importantes
La clasificación de las especies fue del 100\% y se identificaron 17 medidas importantes (Cuadro 1). El análisis de varianza reveló que casi todas las medidas presentaban diferencias significativas $(\mathrm{p}<0.001)$, excepto la altura cefálica. Sin embargo, el análisis de DHS Tukey mostró que sólo 10 medidas permiten diferenciar al menos a una especie; siendo la posición de la boca, la medida más efectiva en la diferenciación (Cuadro 2).

Los datos morfométricos indicaron que $V$. ufermanni tiene la longitud del hocico más larga y la longitud de la mandíbula superior más corta; $V$. pearsei posee los valores más bajos de la longitud predorsal; $V$. intermedia tiene más ancha la cabeza y el cuerpo. La longitud de las aletas dorsal y anal es menor en $V$. intermedia y $V$. pearsei. La aleta caudal es más corta en $V$. intermedia y más larga en $V$. bifasciata y $V$. synspila. La aleta pélvica es más corta en $V$. argentea y $V$. intermedia. El ángulo de la posición bucal es menor en $V$. pearsei y mayor en $V$. argentea (Cuadro 3 ).

A partir de las distancias cuadráticas de Mahalanobis se encontraron diferencias significativas entre todos las cíclidos analizados $(\mathrm{p}<0.0001)$. El dendrograma muestra 2 grupos, en el primero están $V$. pearsei y $V$. intermedia (I); en el segundo, las 4 especies restantes, de las cuales $V$. synspila y $V$. bifasciata muestran la mayor similitud (II) (Fig. 3).

El análisis de discriminantes de la morfometría geométrica mostró que los 2 primeros ejes son estadísticamente diferentes y que existen diferencias significativas entre la forma de los cíclidos (Lambda de Wilk's $=0.0003$, F $(130,443)=14.267, p<0.00001)$. La primera función canónica explicó el $46.4 \%$ de la varianza, mientras que la segunda el $27.7 \%$. El porcentaje de clasificación fue del $98.3 \%$ debido a que un organismo de $V$. bifasciata y otro de $V$. synspila estuvieron mal clasificados.

El análisis también muestra que las especies se separan notablemente. En la región positiva de la primera función se ubican $V$. argentea, $V$. bifasciata, $V$. synspila y parte de $V$. ufermanni, mientras que hacia la parte negativa están el resto de los taxones. En la segunda función, en la parte positiva principalmente, se separa $V$. ufermanni; en cambió en la negativa tienden a ubicarse los ejemplares de $V$. intermedia y $V$. argentea. Se diferenciaron 4 patrones morfológicos, que corresponden a los organismos de $V$. argentea, V. intermedia, V. pearsei y V. ufermanni (Fig. 4).

Las gradillas de deformación de estas especies, muestran que los cíclidos tienen mayor variación en la región cefálica, en la posición de las marcas 1, 10, 11 y 13 con respecto a la configuración promedio (Fig. 1). Las deformaciones de $V$. argentea muestran que posee la cabeza más larga, el ojo en posición anterior y la aleta anal más larga (8) (Fig. 5a). Vieja intermedia (Fig. 5b) tiene la boca en posición más dorsal, como en $V$. pearsei que además 
Cuadro 2. Medidas discriminantes y los valores de $\mathrm{F}$ obtenidos del análisis de varianza en las especies de Vieja con $\mathrm{P}<0.001$, $\mathrm{n}=20$

\begin{tabular}{|c|c|c|}
\hline Medida & $F$ & Grupos o especies diferenciados \\
\hline Longitud del hocico & 24.918 & V. ufermanni \\
\hline Longitud de la mandíbula superior & 32.931 & V. ufermanni \\
\hline Ancho de la cabeza & 20.438 & V. intermedia \\
\hline Longitud predorsal & 25.709 & V. pearsei \\
\hline Ancho del cuerpo & 13.708 & V. intermedia \\
\hline Longitud de la aleta dorsal & 14.298 & $V$. intermedia y $V$. pearsei \\
\hline Longitud de la aleta anal & 120.893 & $V$. intermedia y $V$. pearsei \\
\hline Longitud de la aleta caudal & 64.163 & $V$. intermedia; $V$. synspila y $V$. bifasciata \\
\hline Longitud de la aleta pélvica & 21.086 & $V$. intermedia y $V$. argentea \\
\hline Posición de la boca & 149.586 & $V$. pearsei; $V$. synspila; $V$. bifasciata y $V$. intermedia; $V$. ufermanni; $V$. argentea \\
\hline
\end{tabular}

Cuadro 3.Valores máximos, mínimos y promedios de las medidas discriminantes en las especies estudiadas de Vieja, expresadas en porcentaje con respecto a la longitud estándar

\begin{tabular}{|c|c|c|c|c|c|c|}
\hline Medida & $\begin{array}{c}V . \\
\text { argentea }\end{array}$ & $\begin{array}{c}V . \\
\text { bifasciata }\end{array}$ & $\begin{array}{c}V . \\
\text { intermedia }\end{array}$ & $\begin{array}{c}V . \\
\text { pearsei }\end{array}$ & $\begin{array}{c}V . \\
\text { synspila }\end{array}$ & $\begin{array}{c}V . \\
\text { ufermanni }\end{array}$ \\
\hline Longitud del hocico & $\begin{array}{c}12.2-16.5 \\
(14.7)\end{array}$ & $\begin{array}{c}13.7-17.4 \\
(15.3)\end{array}$ & $\begin{array}{c}13.2-17.4 \\
(15.4)\end{array}$ & $\begin{array}{l}12.6-16.6 \\
(14.7)\end{array}$ & $\begin{array}{l}13.8-17.1 \\
(15.6)\end{array}$ & $\begin{array}{c}16.6-19.6 \\
(18.1)\end{array}$ \\
\hline $\begin{array}{l}\text { Longitud de la } \\
\text { mandíbula superior }\end{array}$ & $\begin{array}{l}9.4-13.2 \\
\quad(11.0)\end{array}$ & $\begin{array}{l}9.1-11.0 \\
\quad(9.7)\end{array}$ & $\begin{array}{l}9.8-10.6 \\
\quad(10.8)\end{array}$ & $\begin{array}{l}9.6-12.0 \\
\quad(10.6)\end{array}$ & $\begin{array}{l}8.9-10.8 \\
\quad(9.5)\end{array}$ & $\begin{array}{l}8.3-9.7 \\
(9.0)\end{array}$ \\
\hline Ancho de la cabeza & $\begin{array}{l}17.0-21.1 \\
\quad(18.3)\end{array}$ & $\begin{array}{l}17.0-19.7 \\
(18.5)\end{array}$ & $\begin{array}{c}19.4-22.3 \\
(20.5)\end{array}$ & $\begin{array}{l}17.0-20.1 \\
\quad(18.3)\end{array}$ & $\begin{array}{l}18.0-20.6 \\
(19.2)\end{array}$ & $\begin{array}{l}17.3-19.8 \\
\quad(18.6)\end{array}$ \\
\hline Longitud predorsal & $\begin{array}{c}39.6-45.3 \\
(42.2)\end{array}$ & $\begin{array}{c}38.1-42.3 \\
\quad(40.2)\end{array}$ & $\begin{array}{c}38.1-42.3 \\
(40.1)\end{array}$ & $\begin{array}{c}35.9-42.0 \\
(38.3)\end{array}$ & $\begin{array}{c}37.9-41.8 \\
(39.9)\end{array}$ & $\begin{array}{l}38.6-43.3 \\
\quad(41.4)\end{array}$ \\
\hline Ancho del cuerpo & $\begin{array}{l}16.8-21.2 \\
\quad(18.3)\end{array}$ & $\begin{array}{l}16.0-19.9 \\
(18.3)\end{array}$ & $\begin{array}{c}18.8-22.0 \\
(20.6)\end{array}$ & $\begin{array}{l}15.0-20.6 \\
(18.0)\end{array}$ & $\begin{array}{l}17.6-20.9 \\
(19.1)\end{array}$ & $\begin{array}{l}15.9-21.3 \\
\quad(19.2)\end{array}$ \\
\hline $\begin{array}{l}\text { Longitud de la aleta } \\
\text { dorsal }\end{array}$ & $\begin{array}{l}58.8-64.8 \\
(61.0)\end{array}$ & $\begin{array}{c}58.5-63.9 \\
(61.5)\end{array}$ & $\begin{array}{c}53.9-60.3 \\
(58.1)\end{array}$ & $\begin{array}{c}54.0-61.8 \\
(59.5)\end{array}$ & $\begin{array}{c}58.8-64.2 \\
(61.0)\end{array}$ & $\begin{array}{l}58.4-63.2 \\
\quad(61.2)\end{array}$ \\
\hline $\begin{array}{l}\text { Longitud de la aleta } \\
\text { anal }\end{array}$ & $\begin{array}{l}24.3-28.5 \\
\quad(25.8)\end{array}$ & $\begin{array}{l}21.5-28.4 \\
(26.2)\end{array}$ & $\begin{array}{c}18.7-22.5 \\
(20.7)\end{array}$ & $\begin{array}{c}18.6-22.3 \\
(20.8)\end{array}$ & $\begin{array}{l}23.7-27.9 \\
(26.1)\end{array}$ & $\begin{array}{l}21.2-25.3 \\
\quad(23.1)\end{array}$ \\
\hline $\begin{array}{l}\text { Longitud de la aleta } \\
\text { caudal }\end{array}$ & $\begin{array}{l}27.2-33.3 \\
\quad(29.8)\end{array}$ & $\begin{array}{c}29.6-34.5 \\
(31.6)\end{array}$ & $\begin{array}{c}23.0-28.0 \\
(25.7)\end{array}$ & $\begin{array}{c}26.7-31.3 \\
\quad(29.4)\end{array}$ & $\begin{array}{c}29.4-34.4 \\
(31.5)\end{array}$ & $\begin{array}{l}26.9-30.6 \\
\quad(29.3)\end{array}$ \\
\hline $\begin{array}{l}\text { Longitud de la aleta } \\
\text { pélvica }\end{array}$ & $\begin{array}{l}22.2-28.6 \\
(25.9)\end{array}$ & $\begin{array}{c}25.8-32.8 \\
(29)\end{array}$ & $\begin{array}{c}22.3-27.1 \\
(24.4)\end{array}$ & $\begin{array}{l}23.6-33.1 \\
\quad(28.4)\end{array}$ & $\begin{array}{l}23.3-35.5 \\
\quad(29.7)\end{array}$ & $\begin{array}{l}23.8-31.8 \\
\quad(29.0)\end{array}$ \\
\hline Posición de la boca & $\begin{array}{c}31^{\circ}-39^{\circ} \\
\left(35.3^{\circ}\right)\end{array}$ & $\begin{array}{c}22^{\circ}-29^{\circ} \\
\left(26.1^{\circ}\right)\end{array}$ & $\begin{array}{c}20^{\circ}-29^{\circ} \\
\left(26.2^{\circ}\right)\end{array}$ & $\begin{array}{c}17^{\circ}-25^{\circ} \\
\left(18.9^{\circ}\right)\end{array}$ & $\begin{array}{c}19^{\circ}-27^{\circ} \\
\left(24.0^{\circ}\right)\end{array}$ & $\begin{array}{c}28^{\circ}-36^{\circ} \\
\left(30.6^{\circ}\right)\end{array}$ \\
\hline
\end{tabular}




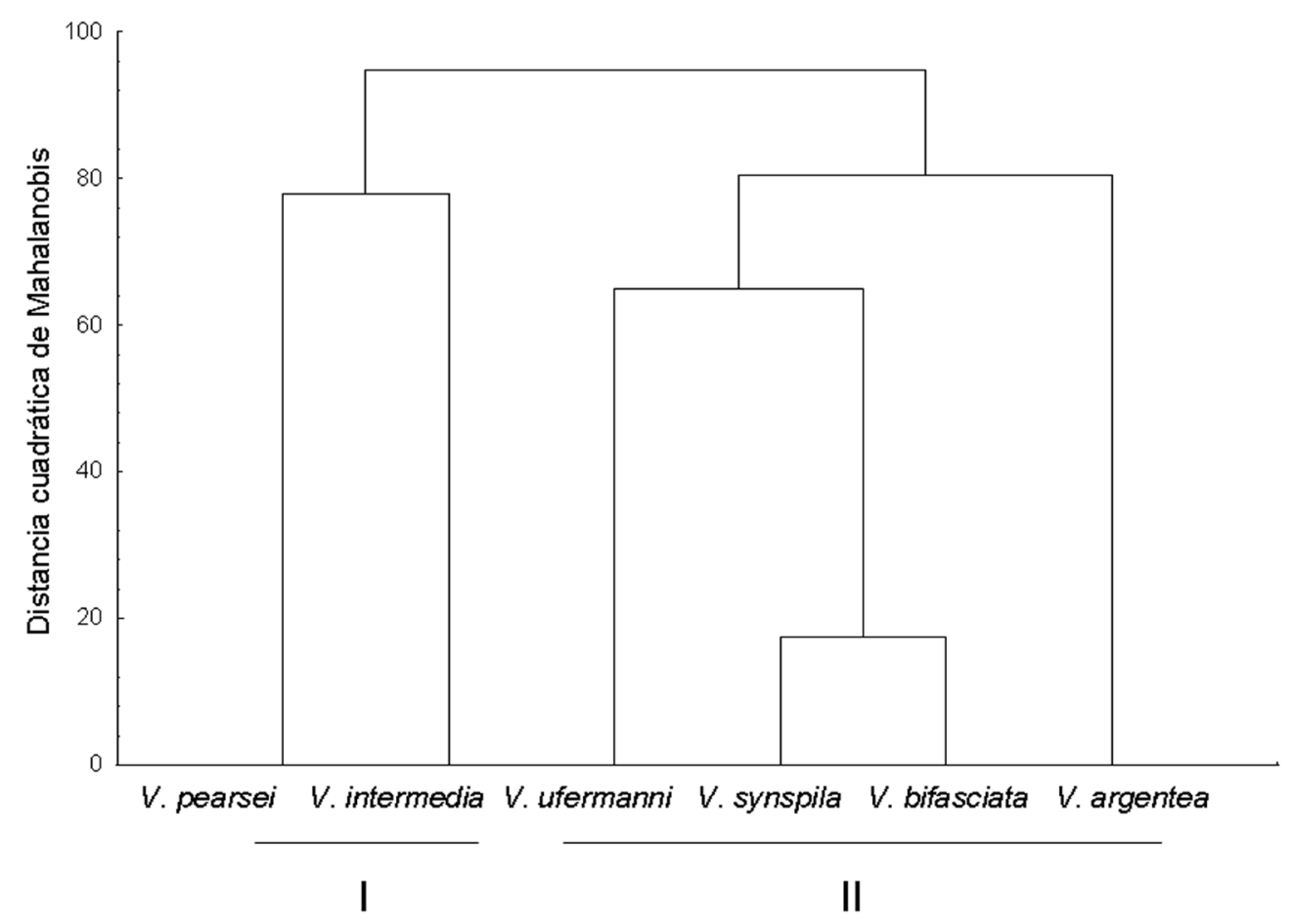

Figura 3. Dendrograma de las especies de Vieja obtenido con las medidas tradicionales a partir del método de agrupamiento UPGMA.

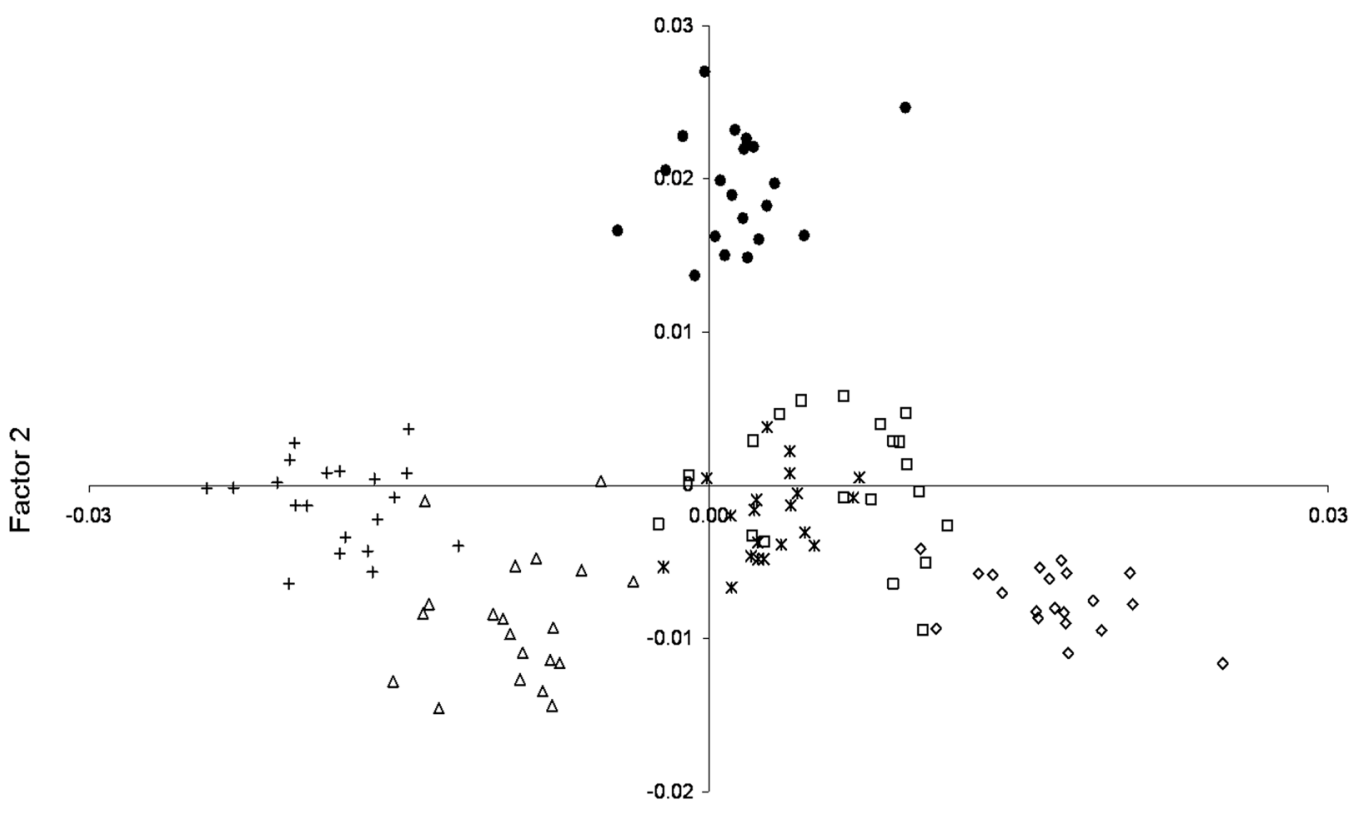

Factor 1

$\diamond V$. argentea $\square$ V. bifasciata $\Delta V$. intermedia $+V$. pearsei $* V$. synspila $\bullet V$. ufermanni

Figura 4. Análisis de discriminantes usando la morfología geométrica de las especies de Vieja. 


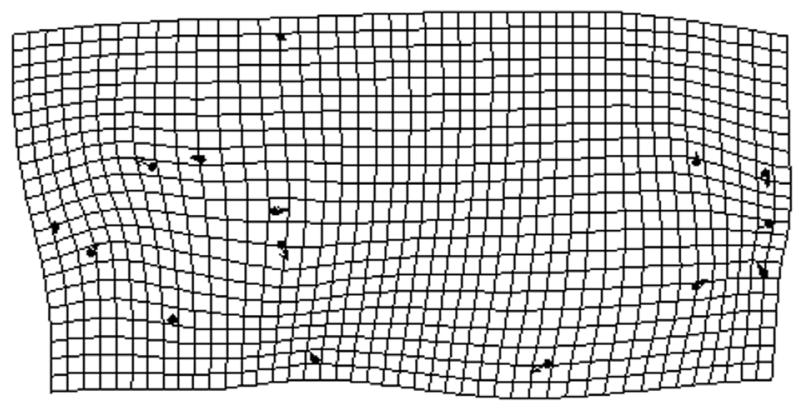

a) Vieja argentea

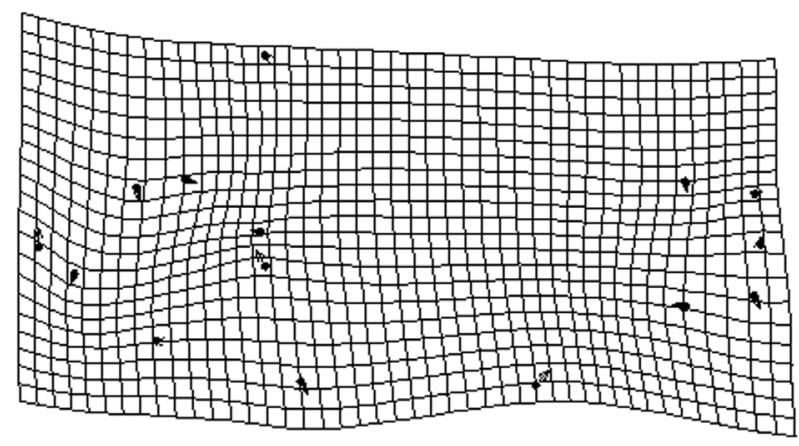

c) Vieja pearsei

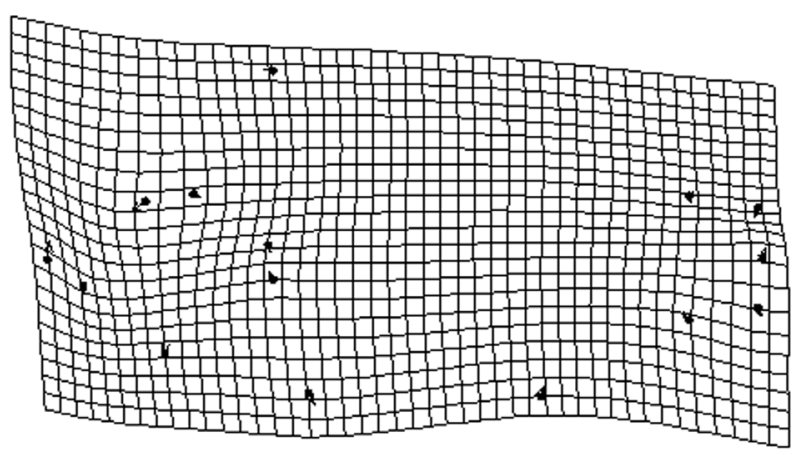

b) Vieja intermedia

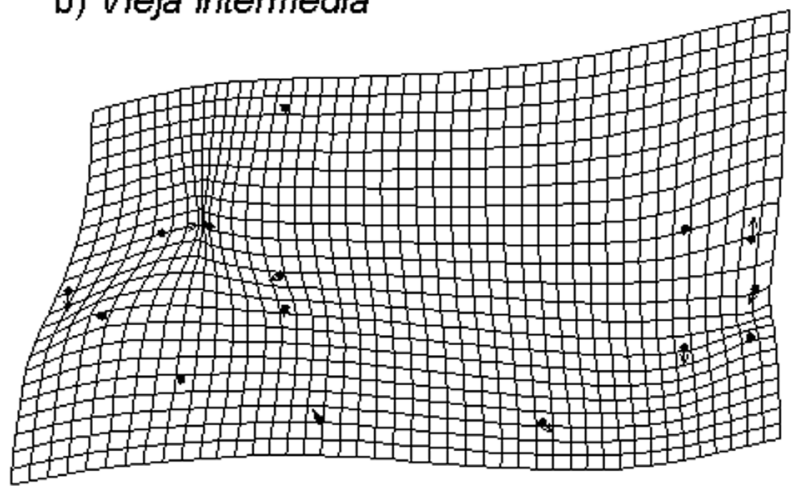

d) Vieja ufermanni

Figura 5. Gradillas de deformación promedio de las especies de Vieja a partir del análisis discriminante. Las imágenes han sido exageradas 3 veces para tener una mejor observación de las diferencias morfológicas.

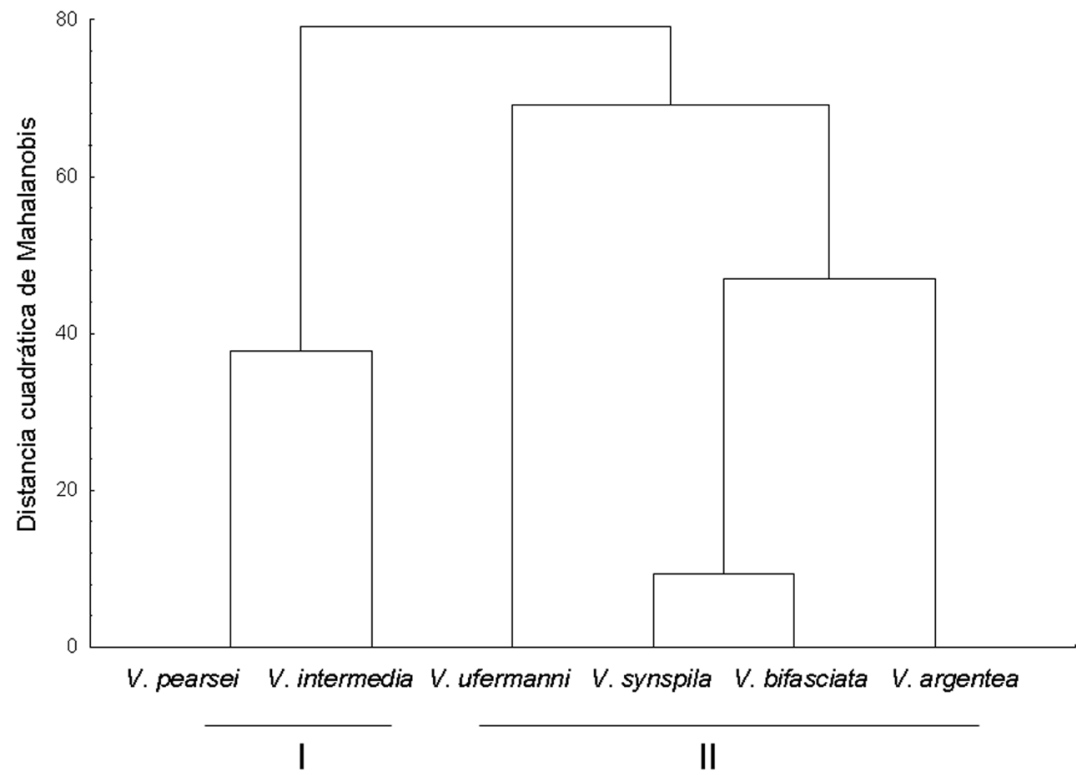

Figura 6. Dendrograma de las especies de Vieja obtenido a partir de las distancias cuadráticas de Mahalanobis con las deformaciones parciales. 
tiene la aleta anal corta y el ojo en posición más ventral (Fig. 5c). Vieja ufermanni tiene la mayor deformación respecto al promedio, relacionada con la posición del ojo y la boca, y en el pedúnculo caudal ( 4,5 y 9) (Fig. $5 d$ ).

La distancia cuadrática de Mahalanobis indicó que existen diferencias significativas entre los cíclidos $(\mathrm{p}<0.0001)$. El dendrograma muestra los mismos 2 grupos, en este caso las relaciones de similitud son más estrechas, sobretodo entre $V$. bifasciata y $V$. synspila; además se observa la separación de $V$. ufermanni en el grupo II (Fig. 6).

\section{Discusión}

El análisis de morfometría tradicional mostró que las especies de Vieja en la cuenca del Usumacinta son entidades taxonómicas diferenciadas morfológicamente, que pueden distinguirse con 10 medidas relacionadas con la longitud de las aletas, forma de la cabeza y ancho del cuerpo. Estas medidas pueden emplearse para la determinación taxonómica de estos cíclidos, lo cual facilitará el reconocimiento de ejemplares procedentes de colecciones. En especial servirán para diferenciar a $V$. ufermanni, la cual no está incluida en las claves más actualizadas y en cuya diagnosis se considera principalmente el patrón de coloración (Miller et al., 2005). Además, las medidas podrán complementar los caracteres usados en la clave de determinación, cuando las características merísticas y de coloración no sean del todo satisfactorias.

Aunque se reconocieron 10 medidas discriminantes, la posición de la boca fue la más efectiva en la diferenciación de estos cíclidos. Esta medida puede obtenerse fácilmente (Winemiller, 1991), por ello se sugiere su uso en la determinación práctica de las especies, en especial para $V$. bifasciata y $V$. synspila. Estos taxones son simpátricos y su determinación a partir de material fijado es difícil porque se basa en la presencia de bandas longitudinales, la altura del cuerpo o la longitud de las aletas pares.

Es importante señalar que el análisis morfométrico se restringió a organismos adultos; es probable que las medidas discriminantes cambien en los jóvenes, debido a que los peces manifiestan cambios corporales en su crecimiento relacionados con el hábitat y la dieta (Meyer, 1987). Se sabe que $V$. ufermanni manifiesta cambios en la posición de la boca; en las tallas pequeñas se ubica dorsalmente y en los adultos hacia la región ventral. Estas modificaciones se asocian al cambio de hábitat de los adultos hacia zonas pelágicas y al consumo de alimento bentónico (SoriaBarreto, 2009). Considerando lo anterior, es necesario conocer cómo cambian las características morfométricas en el resto de los cíclidos, si las diferencias se mantienen, y por ello se deben tomar con cautela.

Se ha sugerido que por la forma del cuerpo, las especies de Vieja se pueden dividir en 2 grupos: organismos con cuerpos altos y robustos; y aquellos con cuerpos más alargados y delgados (Conkel, 1993; Miller et al., 2005). En este trabajo, el análisis de la morfometría geométrica evidenció que los taxones analizados podrían pertenecer al primer grupo, debido a que las deformaciones morfológicas no muestran variaciones en la altura corporal, más bien se diferencian en la posición de la boca y del ojo y, en menor grado, en la altura del pedúnculo caudal.

A pesar de que en $V$. intermedia, $V$. pearsei y $V$. ufermanni se observaron las mayores diferencias morfológicas tradicionales y en la morfometría geométrica. se considera que estas características no son suficientes para ubicarlas en otro género como se hizo anteriormente (Hulsey et al., 2004; Concheiro et al., 2007). Es necesario analizar todas las especies de Vieja, empleando no sólo características morfométricas, se recomienda incluir las merísticas, osteológicas y moleculares, que en conjunto proporcionen una mejor aproximación de las relaciones filogenéticas de estos cíclidos.

En cambio, los análisis mostraron una gran similitud morfológica entre $V$. bifasciata y $V$. synspila Estos peces muestran características parecidas y se diferencian por el patrón de coloración (Miller et al., 2005). No obstante, en este trabajo se evidenció que $V$. synspila tiene la boca ubicada en posición ligeramente más ventral que $V$. bifasciata, que es un carácter importante en su diferenciación, aún así son cíclidos que ameritan el estudio de otras características morfológicas.

Se considera que los resultados de ambos métodos morfométricos son complementarios. Las formas obtenidas en la morfometría geométrica concuerdan con la diferenciación encontrada con las medidas tradicionales; la primera muestra la variación corporal en conjunto y las últimas permitieron identificar características discriminantes entre los cíclidos. Un caso semejante se observó con las especies de Amphilophus en Centroamérica; empleando ambos métodos se obtuvo una mejor aproximación para conocer la variación morfológica interespecífica (Parsons et al., 2003). En cambio, en otros estudios la morfometría geométrica es más efectiva que la tradicional, como ocurrió con las poblaciones del cíclido Tropheus moorii del lago Tanganyika (Maderbacher et al., 2008) y con los morfos del cíclido mexicano Herichthys minckleyi (Trapani, 2003).

Los resultados indican que las especies de Vieja tienen características corporales diferenciales, principalmente en la región cefálica. Por lo cual se sugiere analizar estructuras y características relacionadas con la captura y procesamiento del alimento, como los huesos de la región 
hiomandibular y en especial el aparato faríngeo, el cual se ha considerado como un elemento importante en la evolución y diversificación de los cíclidos. También es recomendable asociar las características morfológicas y osteológicas con la alimentación de las especies, con el objetivo de conocer si el reparto de recursos puede ser un factor determinante que facilite su coexistencia.

\section{Agradecimientos}

Este trabajo forma parte de la tesis doctoral de la primera autora y fue financiado por Consejo Nacional de Ciencia y Tecnología (CONACyT) con Apoyo Integral para la Formación de Doctores en Ciencias (Posgrado de ECOSUR). En la Colección de Peces (ECOSC) de El Colegio de la Frontera Sur (ECOSUR), Unidad San Cristóbal, se encuentra albergado el material científico que fue analizado.

\section{Literatura citada}

Adams, D. C., F. J. Rohlf y D. E. Slice. 2004. Geometric morphometrics: ten years of progress following the 'revolution'. Italian Journal of Zoology 71:5-16.

Allgayer, R. 1989. Révision et redescription du genre Theraps Günther, 1862. Description de deux espèces nouvelles du Mexique (Pisces, Perciformes, Cichlidae). Revue Française des Cichlidophiles 90bis:4-30.

Allgayer, R. 2002. Vieja ufermanni sp. nov., un Cichlidé nouveau du bassin du Río Usumacinta et du Río de la Pasión, Amérique centrale (Pisces: Perciformes). L'an Cichlidé, 2:14-17.

Catena, A., M. M. Ramos y H. M. Trujillo. 2003. Análisis multivariado. Un manual para investigadores. Biblioteca Nueva, Madrid. 413 p.

Chávez-Lomelí, M. O., A. E. Mattheeuws y M. H. Pérez-Vega. 1988. Biología de los peces del río San Pedro en vista de determinar su potencial para la piscicultura. Instituto Nacional de Investigaciones sobre Recursos Bióticos, Villahermosa, Tabasco. $222 \mathrm{p}$.

Concheiro, P. G. A., O. Rícan, G. Ortí, E. Birmingham, I. Doadrio y R. Zardoya. 2007. Phylogeny and biogeography of 91 species of heroine cichlids (Teleostei: Cichlidae) based on sequences of the cytochrome b gene. Molecular Phylogenetics and Evolution 43:91-110.

Conkel, D. 1993. Cichlids of North and Central America. T.F.H., Neptune City, NJ. 64 p.

Crisci, J. V. y M. F. López Armengol. 1983. Introducción a la teoría y práctica de la taxonomía numérica. OEA, Washington, D.C. $132 \mathrm{p}$.
Eschmeyer, W. N. y R. Fricke. (eds.), 2010. Catalog of Fishes electronic version (15 January 2010). http://research. calacademy.org/ichthyology/catalog/fishcatmain.asp

Froese, R. y D. Pauly. (eds.). 2010. FishBase.World wide web electronic publication. www.fishbase.org, version (01/2010); 20.II.2010

González-Díaz, A. A., R. M. Quiñones, J. Velázquez-Martínez y R. Rodiles-Hernández. 2008. Fishes of La Venta River in Chiapas, Mexico. Zootaxa 1685:47-54.

Hulsey, C. D., F. J. García de León, Y. Sánchez, D. A. Hendrickson y T. J. Near. 2004. Temporal diversification of Mesoamerican cichlid fishes across a major biogeographic boundary. Molecular Phylogenetics and Evolution 31:754-464.

INE (Instituto Nacional de Ecología). 2000. Programa de manejo de Reserva de la Biosfera Montes Azules, México. Instituto Nacional de Ecología, SEMARNAP, México, D.F. 255 p.

Konings, A. 1989. Cichlids from Central America. T. F. H., Neptune City, New Jersey. 222 p.

Kullander, S. O. 1983. A revision of the South American cichlid genus Cichlasoma (Teleostei:Cichlidae). Swedish Museum of Natural History, Stockholm. 296 p.

Kullander, S. O. 2003. Family Cichlidae (Cichlids). In Check list of the freshwater fishes of South and Central America, R. E. Reis, S. O. Kullander, y C. J. Jr. Ferraris (orgs.). Edipucrs, Porto Alegre. p. 605-654.

Maderbacher, M., C. Bauer, J. Herler, L. Postl, L. Makasa y C. Sturmbauer. 2008. Assessment of traditional versus geometric morphometrics for discriminating populations of the Tropheus moorii species complex (Teleostei: Cichlidae), a Lake Tanganyika model for allopatric speciation. Journal of Zoological Systematics and Evolutionary Research 46:153-161.

Meyer, A. 1987. Phenotypic plasticity and heterochrony in Cichlasoma managuense (Pisces, Cichlidae) and their implications for speciation in cichlid fishes. Evolution 41:1357-1369.

Miller, R. R. 1986. Composition and derivation of the freshwater fish fauna of México. Anales de la Escuela Nacional de Ciencias Biológicas [México] 30:121-153.

Miller, R. R., W. L. Minckley y S. M. Norris. 2005. Freshwater fishes of México. The Chicago University Press, Illinois. 490 p.

Parsons, K. J., B. W. Robinson y T. Hrbek. 2003. Getting into shape: an empirical comparison of traditional truss-based morphometric methods with a newer geometric method applied to New World cichlids. Environmental Biology of Fishes 67:417-431.

Regan, C. T. 1905. A revision of the fishes of the American cichlid genus Cichlosoma and of the allied genera. Annals and Magazine of Natural History 16:60-77, 225-243, 316$341,433-445$.

Rícan, O., R. Zardoya e I. Doadrio. 2008. Phylogenetic relationships of Middle American cichlids (Cichlidae, Heroini) based on combined evidence from nuclear genes, 
mtDNA, and morphology. Molecular Phylogenetics and Evolution 49:941-957.

Rodiles-Hernández, R., Díaz-Pardo y J. Lyons. 1999. Patterns in the species diversity and composition of the fish community of the Lacanja River, Chiapas, Mexico. Journal of Freshwater Ecology 14:455-468.

Roe, K. J., D. Conkel y C. Liderad. 1997. Molecular systematics of middle american cichlid fishes and evolution of trophictypes in 'Cichlasoma (Amphilophus)' and 'C. (Thorichthys)'. Molecular Phylogenetics and Evolution 17:366-376.

Rohlf, F. J. 1999. Shape statistics: Procrustes superimpositions and tangent spaces. Journal of Classification 16:197-223.

Rohlf, F. J. 2004. tpsDig, digitize landmarks and outlines, version 2.0. Department of Ecology and Evolution, State University of New York at Stony Brook.

Sheets, H. D. 2001. CoordGen6f, CVAGen6m, PCAGen6n, Regress6k, Tmorphgen6a disponible en IMP. Dept. of Physics, Canisius College, Buffalo, New York 14208,
Dept. of Geology, SUNY at Buffalo, Buffalo New York. 14260. http://www.canisius,edu/ sheets/morphsoft.html; 20.II.2010.

Soria-Barreto, M. 2009. Ecomorfología de los cíclidos en la selva lacandona (REBIMA), Chiapas, México. Tesis, doctorado El Colegio de la Frontera Sur, San Cristóbal de Las Casas, Chiapas. 153 p.

StatSoft. 1995. Statistica for Windows (Computer program manual). StatSoft, Tulsa, Oklahoma.

Trapani, J. 2003. Geometric morphometric analysis of body-form variability in Cichlasoma minckleyi, the Cuatro Cienegas cichlid. Environmental Biology of Fishes 68:357-369.

Winemiller, K. O. 1991. Ecomorphological diversification in lowland freshwater fish assemblages from five biotic regions. Ecological Monographs 61:343-365.

Zelditch, M. L., H. D. Swiderski, H. D. Sheets y W. L. Fink. 2004. Geometric morphometrics for biologists: a primer. Elsevier, New York. 443 p. 
